\title{
Application of 3D Whole Body Scanning in Research on Human Body Surface Area
}

\author{
Grzegorz REDLARSKI, Marek KRAWCZUK, Aleksander PALKOWSKI* \\ Gdansk University of Technology, Department of Mechatronics and High Voltage Engineering, \\ Gdansk, Poland
}

DOI: $10.15221 / 17.060 \quad$ http://dx.doi.org/10.15221/17.060

\begin{abstract}
Human body surface area (BSA) is one of the major parameters used in several medical fields. Its heterogeneity caused by individual human characteristics sustains a many-decades-long research on the matter. Today's technology allows to create exact body models in mere seconds. However, an extensive research that includes scanning people with major disfigurements or people suffering from diseases, which often prevent their mobility, requires a specific approach. Here, we present the entire scanning procedure and graphical processing methods used in investigation of changes in BSA in function of different anthropometric parameters. The Artec 3D Eva hand-held scanner has been used as the measurement device. We performed a total of five scans for every subject-four for each limb and one for the main body part. After a series of processing methods, the resulting body model can be further used as an accurate basis for BSA formulae investigation.
\end{abstract}

Keywords: 3D body processing, 3D body scanning, anthropometry, Artec 3D Eva, body surface area, medical sciences

\section{Introduction}

Human body surface area (BSA), the measured or calculated surface area of a human body, is one of the major parameters used in medicine and physiology. Because of being unaffected by abnormal adipose mass, BSA is considered a better indicator of metabolic mass than the body weight or body mass index (BMI). BSA is used as a basis for deciding the course of treatment in a number of medical fields, such as oncology or transplantology. BSA is an established parameter for the calculation of chemotherapy drugs dosage [1], [2], [3], [4], treatment of chronic hepatitis B [5], treatment of burns [6], or for establishing a dosing regimen for antimicrobials [7].

Attempts to measure the BSA date back to 1879 , when Meeh formulated the first available BSA formula [8]. Not long after, the DuBois brothers published their version of the formula introducing height as a variable; they have used coating bandages to measure the exact BSA values in a group of a few patients [9].

In subsequent years of the twentieth and twenty-first centuries, many studies have tried to identify more precise values for the coefficients of the DuBois formula to accurately describe the human BSA. The most recent studies have used precise 3D laser scanning techniques to determine the human BSA with a substantially higher number of scanned subjects (3951 in Yu, Lo, and Chiou [10], 188 in Schlich [11], and 270 in Yu, Lin, and Yang [12]).

Based on the analysis of popular BSA formulae alone, one can see ambiguity concerning their effect [13]. This premise is the basis for an extended BSA analysis initiated by our team. To better understand how BSA values are formed and from which additional parameters are dependent, an extended analysis of human's physiology must have been conducted. However, an extensive research that includes scanning people with major disfigurements or people suffering from diseases, which often prevents their mobility, requires a specific approach. This has forced a mobile approach for scanning people's body surface.

Here, we present the entire procedure of scanning the BSA of chosen subjects. Our approach must have been adapted to fulfill the requirement of mobility and swiftness of scanning. Therefore, not only the scanning equipment was appropriately chosen, but also all supporting equipment and the procedure itself were deliberately designed to support those ideals.

As a result of the activities concerned, we were able to scan with high precision over 200 patients from several medical facilities across Poland. 


\section{Materials and methods}

\subsection{Study design}

The main purpose of the study is to design and eventually improve a whole body scanning procedure. Bearing in mind that the examination will not be conducted in one place, the whole procedure, along with all of the equipment, should be designed to be mobile as much as possible. To further assist scanning of, e.g., disabled people, the procedure should also be swift and cause little to none physical discomfort.

Therefore, the general examination plan is designed as follows. Firstly, to gather possibly useful medical information, the patient's body is thoroughly examined, i.e., most of significant anthropometric measures are collected. Secondly, the whole body 3D scanning is conducted. With a handheld 3D scanner, the whole model is unlikely to be made in one sitting. Therefore, the scanning procedure must be divided into stages, each ensuring maximum comfort for the patient and stabilization for the scanner. Lastly, the raw body scan is subjected to a series of graphical procedures in order to dispose of all graphical artifacts and to prepare the model for further calculations.

The study was approved by Independent Bioethics Commission for Research at Medical University of Gdansk. Written informed participation consent was obtained from all the volunteers taking part in the examination. All experiments presented were carried out in accordance with the approved guidelines.

\subsection{Equipment used}

To obtain most of the significant anthropometric parameters, we have used a seca 201 tape to measure all circumferences, a stiff measuring tape to measure all lengths, and a MENSOR WE200P3 $M(X)$ medical scale (Fig. 1a) to measure body height and weight.

For reasons such as long scanning time or stability issues, the main body scan is probably the hardest stage to efficiently design. Nevertheless, we propose the following simple procedure. To facilitate the process, a remotely-controlled turntable was constructed (Fig. 1C). The patient is standing on the turntable while being very carefully rotated during the scanning process. Furthermore, the patient is stabilized with two crutches (Fig. 1b) placed under his arms.
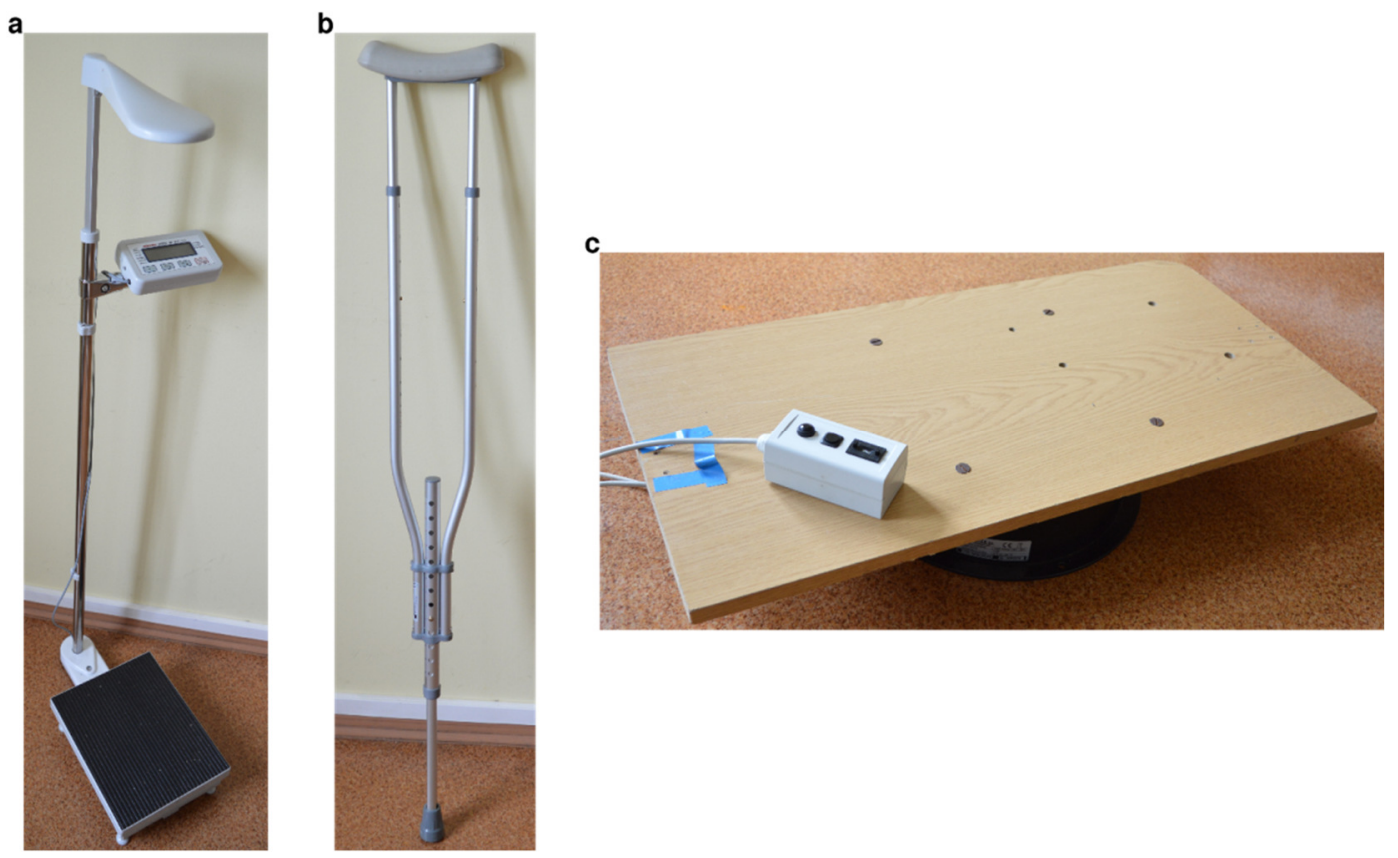

Fig. 1. Supporting equipment used during the measurement and scanning processes. (a) MENSOR WE200P3 $M(X)$ medical scale used to measure body height and weight. (b) One of two crutches used as stabilizers for the body. (c) Remotely-controlled turntable used to facilitate the scanning process.

The scanning device used is the Artec 3D Eva handheld scanner (Fig. 2). The scanner's operation is based on white light irradiation and video camera triangulation, therefore, it is safe for most people, excluding those suffering from epilepsy. The scanner's maximum accuracy of $0.1 \mathrm{~mm}$ allows to produce high-quality 3D models.

Furthermore, a high-end laptop computer is used to gather the scans and carry out all preliminary graphical operations. 


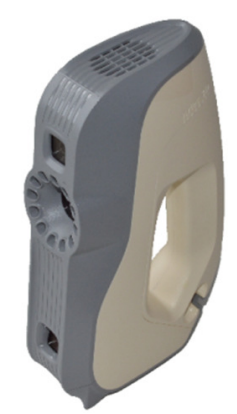

Fig. 2. Artec 3D Eva scanner used as the scanning device.

\subsection{Software used}

Most of the graphical operations are made in the dedicated Artec Studio 11 Professional software (Fig. 3). The application supports the model-building pipeline right from live construction of the initial body scan to final post-processing operations. However, Artec Studio is not a specialized graphical software, therefore 3D model manipulation is hampered.

Often 3D models produced by the Artec Studio still require several graphical operations for the models to be appropriate for analysis. The models tend to have minor flaws, e.g., small irregularities, protrusions, or breaks. These flaws are fixed using the Autodesk Meshmixer software (Fig. 4), which provides appropriate and easy-to-use sculpting tools.

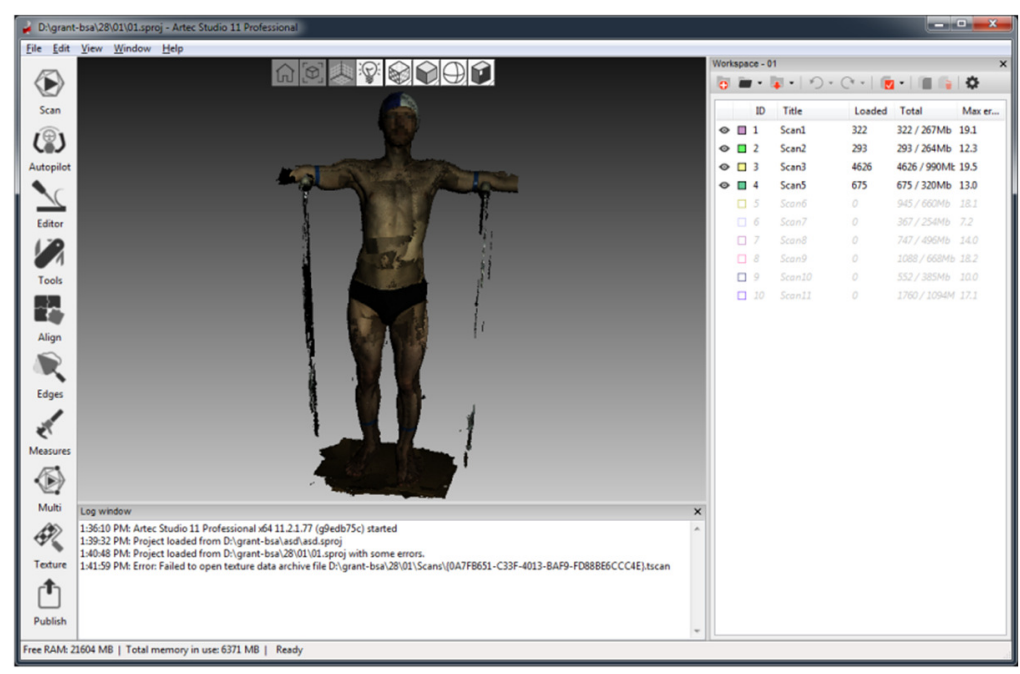

Fig. 3. Artec Studio 11 Professional.

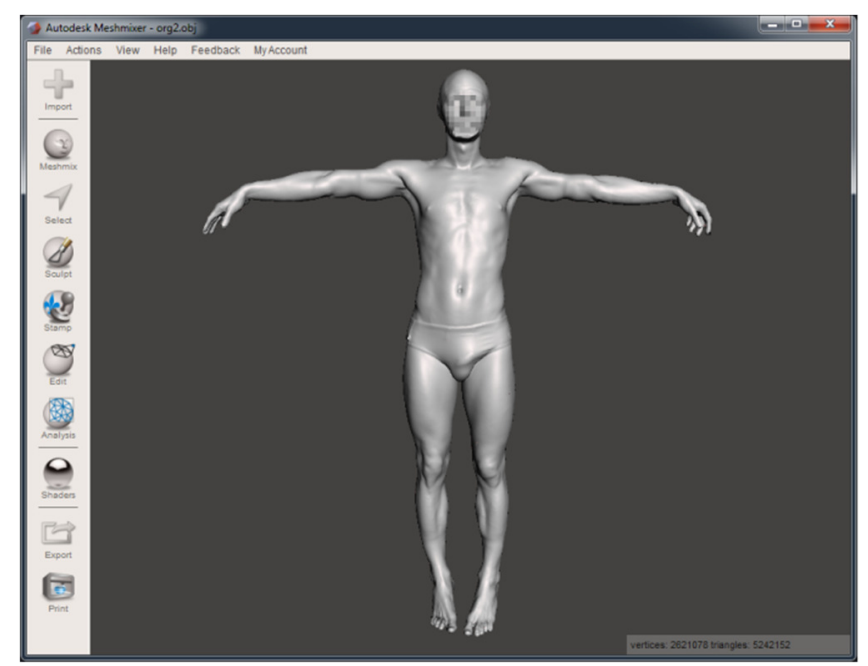

Fig. 4. Autodesk Meshmixer 3.2. 


\section{Results}

\subsection{Basic measurements}

Besides the most important measure gathered-the BSA-we also obtain several anthropometric parameters that describe a patient's body. The goal is to gather a sufficiently big database of anthropometric measures that could be used (aside from height and weight) as a base for BSA estimation.

The additional measures gathered are: head circumference, distance between the outer ends of eyes, neck circumference, chest circumference, waist circumference, hips circumference, height from the ground to the hip, arm circumference, forearm circumference, wrist circumference, distance between the elbow and the tip of the middle finger, arm span, thigh circumference, calf circumference, height from the ground to the knee, and standard European shoe size. Fig. 5 approximately presents the relevant measures.

We also note the age and sex of a subject, which, together with the shoe size, are acquired based on an interview with a volunteer preceding the examination.

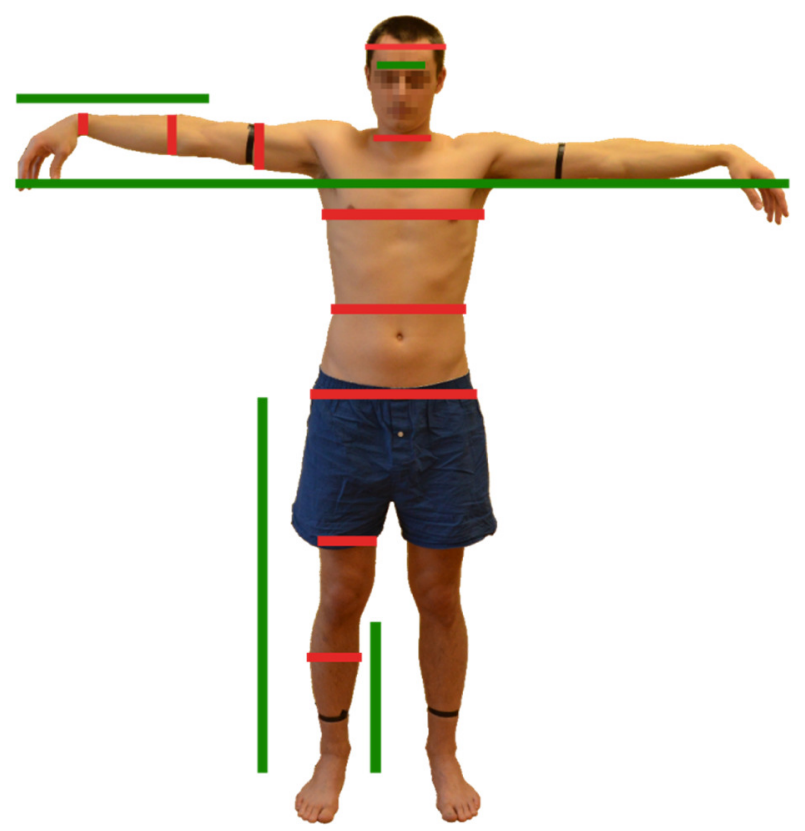

Fig. 5. Human body circumferences (red lines) and lengths (green lines) measured during the study.

\subsection{Whole body 3D scan}

The scanning process is conducted in an upright position and, in cases of severe physical dysfunctions or limb scanning, is conducted seated. We perform a total of five scans for every subject-four for each limb and one for the main body part.

Before the start, we give the subject a swimming cap to cover his hair. This is especially important because the scanner do not handle well surfaces densely covered with hair. We also use tape to mark places where we join all five scans for the final model.

The first scan is made in an upright position stabilized on a turntable (Fig. 6a). Within about 15 minutes the main body scan is being made by carefully covering the body with the scanner device. The next pair of scans are being made seated with one leg outstretched (Fig. 6b), during which we thoroughly scan the feet up to the markers. Means to stabilize the patient are procured on-site, which generally are soft chairs. The last pair of scans concern the hands. A modified tripod is used to stabilize the patient's hand (Fig. $6 \mathrm{c}$ ). The hand scans are also being made up to the arm markers. The previously described four scans take about 3 minutes each to complete.

The final body model is obtained through a series of graphical processing methods. The most of the process is conducted within the Artec Studio software. Firstly, the points cloud obtained from the scan (left side of Fig. 7) is subjected to a global registration process, which normalizes the points locations. Secondly, the normalized point cloud is subjected to a smooth fusion process, resulting in a solid representation of the model. Finally, a series of post-processing methods, such as small-objects filter, hole filling, and smoothing, are used on the solid model to improve its quality. 
For a final touch, the model is imported to the Autodesk Meshmixer software where we fix several minor graphical flaws, e.g., small irregularities, protrusions, or breaks. After the process the final model (right side of Fig. 7) is imported back to the Artec Studio software.

The BSA value is calculated by the Artec Studio software based on surface of the final 3D model. The Measures-Sections tool is used to facilitate the process.
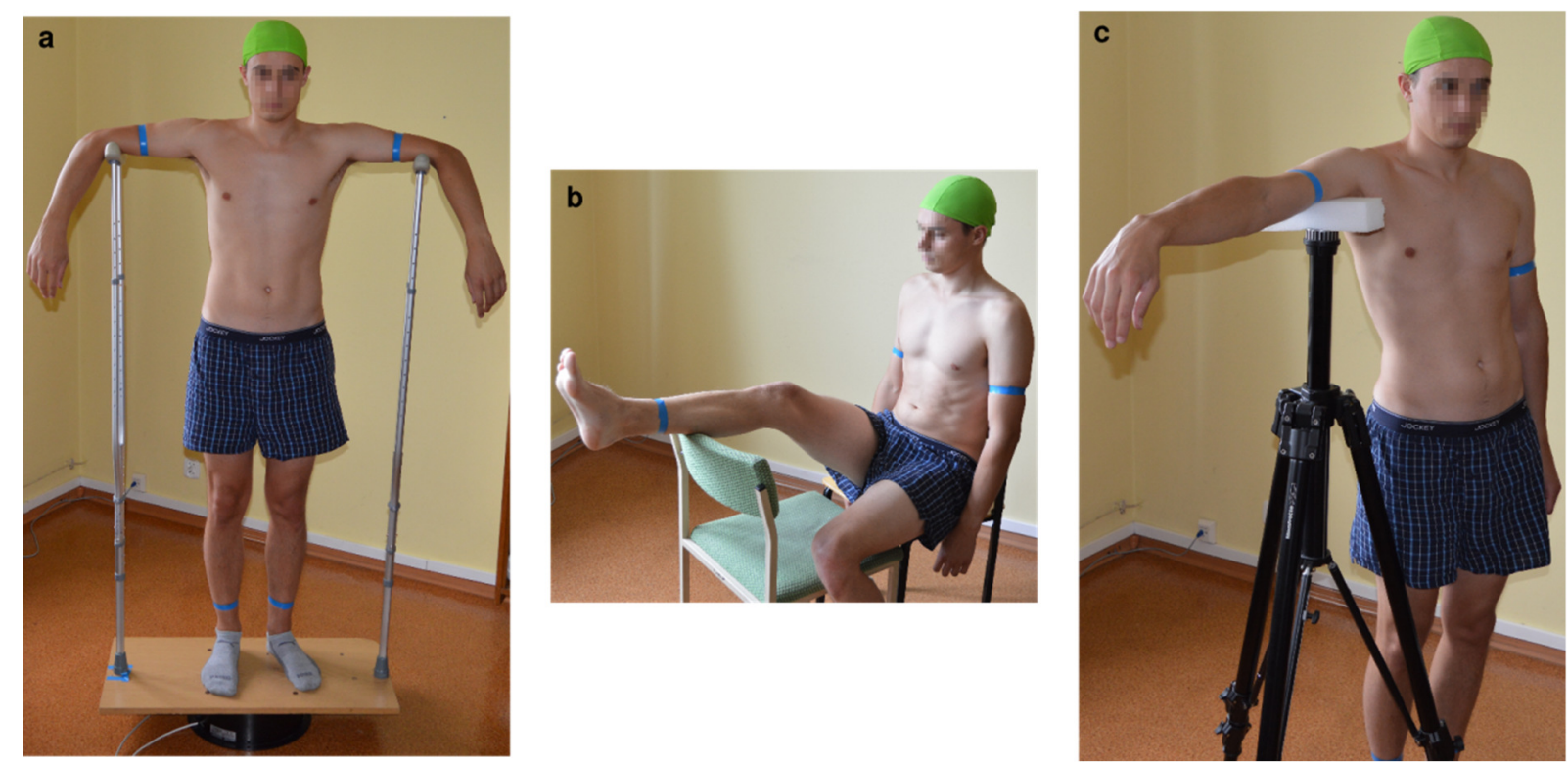

Fig. 6. Body scanning stages. (a) Upright position stabilized on a turntable during the main body scan. (b) Seated position with an outstretched leg during foot scan. (c) Upright position with an outstretched hand during hand scan.

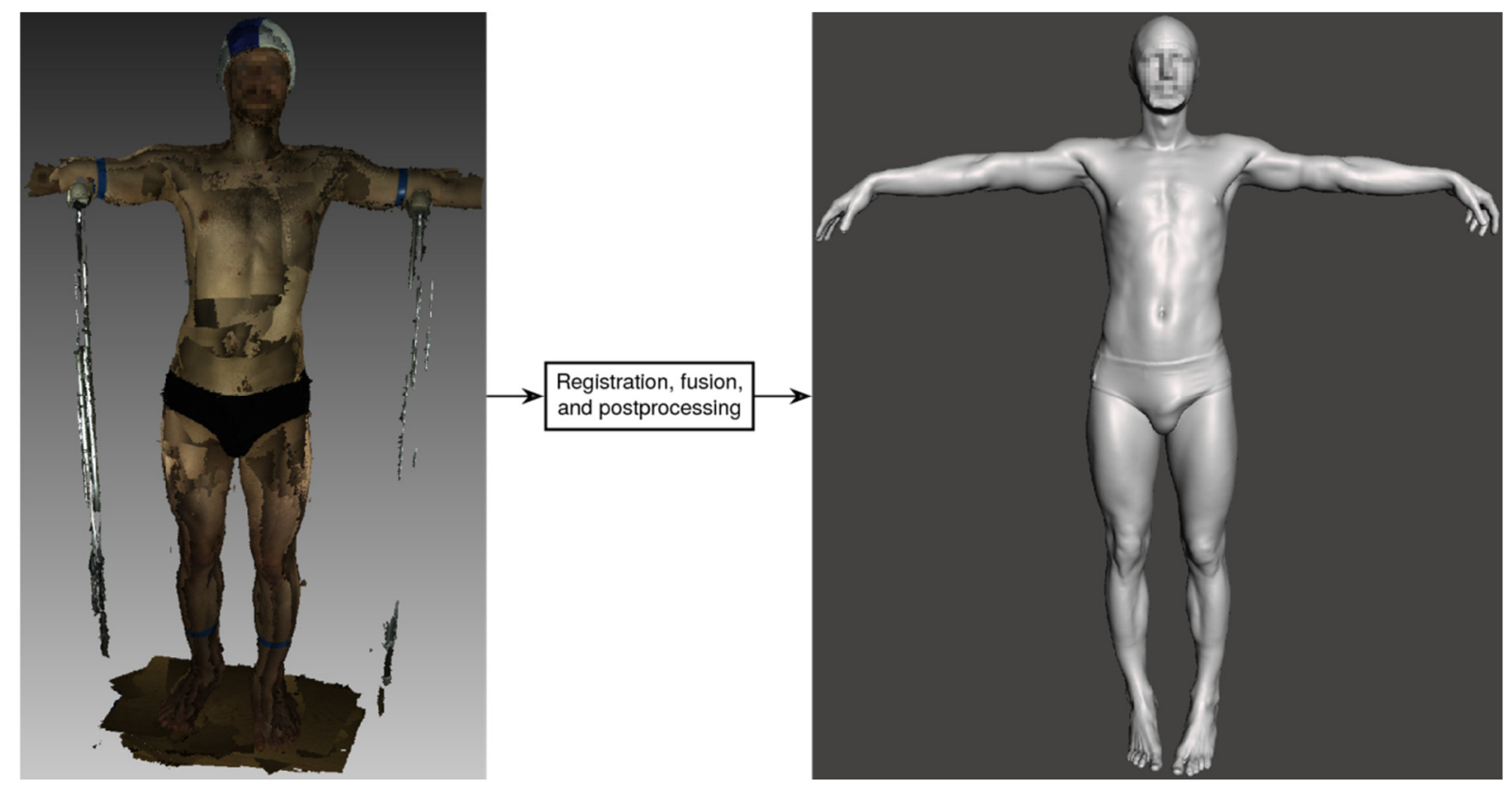

Fig. 7. 3D model processing from a raw scan (on the left) to a final polished body model (on the right).

\section{Conclusions}

The paper presents a whole body scanning procedure designed for the analysis of human BSA. The research conducted includes scanning people with major disfigurements or people suffering from diseases, which often prevent their mobility. Therefore, the design required a specific approach.

By using the Artec 3D Eva handheld scanner and several other pieces of equipment modified for the task, we were able to acquire over 200 3D body models from several medical facilities. 
The scanner's maximum accuracy of $0.1 \mathrm{~mm}$, careful measuring process, and the software used allowed to obtain BSA values to the nearest hundred millionth part of a meter. The collected data can be further used to develop advanced BSA estimation methods.

\section{Acknowledgments}

This work was supported by funds from the Polish National Science Centre (2014/15/B/NZ7/01018).

\section{References}

[1] T. Kouno, N. Katsumata, H. Mukai, M. Ando and T. Watanabe, "Standardization of the Body Surface Area (BSA) Formula to Calculate the Dose of Anticancer Agents in Japan", Jpn. J. Clin. Oncol, Vol.33, 2003, pp. 309-313.

[2] A. Gerina-Berzina, U. Vikmanis, U. Teibe and S. Umbrashko, "Anthropometric measurements of the body composition of cancer patients determine the precise role of the body surface area and the calculation of the dose of chemotherapy", Pap. Anthropol, Vol.21, 2012.

[3] S.D. Baker et al., "Role of Body Surface Area in Dosing of Investigational Anticancer Agents in Adults, 1991-2001", J. Natl. Cancer Inst, Vol.94, 2002, pp. 1883-1888, http://dx.doi.org/10.1093/jnci/94.24.1883.

[4] J.J. Sacco, J. Botten, F. Macbeth, A. Bagust and P. Clark, "The Average Body Surface Area of Adult Cancer Patients in the UK: A Multicentre Retrospective Study", PLoS One, Vol.5, 2010, p. e8933, http://dx.doi.org/10.1371/journal.pone.0008933.

[5] M. Nakamuta et al., "Relationship between body surface area and ALT normalization after long-term lamivudine treatment", World J Gastroenterol, Vol.11, 2005, pp. 6948-6953, http://dx.doi.org/10.3748/wjg.v11.i44.6948.

[6] B.D. Reading and B. Freeman, "Simple formula for the surface area of the body and a simple model for anthropometry", Clin Anat, Vol.18, 2005, pp. 126-130, http://dx.doi.org/10.1002/ca.20047.

[7] M.P. Pai, "Drug Dosing Based on Weight and Body Surface Area: Mathematical Assumptions and Limitations in Obese Adults", Pharmacother J Hum Pharmacol Drug Ther, Vol.32, 2012, pp. 856-868, http://dx.doi.org/10.1002/j.1875-9114.2012.01108.x.

[8] K. Meeh, "Oberflächenmessungen des menschlichen Körpers", Z Biol, Vol.15, 1879, pp. 425-458.

[9] D. Du Bois and E.F. Du Bois, "A formula to estimate the approximate surface area if height and weight be known", in Arch Intern Med, Vol.17, 1916, pp. 863-871.

[10]C.-Y. Yu, Y.-H. Lo and W.-K. Chiou, "The 3D scanner for measuring body surface area: a simplified calculation in the Chinese adult", Appl Ergon, Vol.34, 2003, pp. 273-278, http://dx.doi.org/10.1016/S0003-6870(03)00007-3.

[11]E. Schlich, M. Schumm and M. Schlich, "3D-Body-Scan als anthropometrisches Verfahren zur Bestimmung der spezifischen Körperoberfläche", Ernährungs Umschau, Vol.4, 2010, pp. 178-183.

[12] C.-Y. Yu, C.-H. Lin and Y.-H. Yang, "Human body surface area database and estimation formula", Burns, Vol.36, 2010, pp. 616-629, http://dx.doi.org/10.1016/j.burns.2009.05.013.

[13] G. Redlarski, A. Palkowski and M. Krawczuk, "Body surface area formulae: an alarming ambiguity", Sci Rep, Vol.6, 2016, p. 27966, http://dx.doi.org/10.1038/srep27966. 